Théologiques

Théologiques

\title{
L'expérience de la théologie : corrélation, interruption et recontextualisation
}

\section{Marc Dumas}

Volume 14, numéro 1-2, automne 2006

Les lieux de la théologie aujourd’hui

URI : https://id.erudit.org/iderudit/014314ar

DOI : https://doi.org/10.7202/014314ar

Aller au sommaire du numéro

Éditeur(s)

Faculté de théologie et de sciences des religions, Université de Montréal

ISSN

1188-7109 (imprimé)

1492-1413 (numérique)

Découvrir la revue

Citer cet article

Dumas, M. (2006). L'expérience de la théologie : corrélation, interruption et recontextualisation. Théologiques, 14(1-2), 117-126.

https://doi.org/10.7202/014314ar
Résumé de l'article

L'auteur s'interroge sur la remise en question de la théologie dans ses lieux, qui lui étaient habituellement acquis. En effet, l'université, l'Église et la culture semblent embarrassées par la théologie. Mais cette dernière historiquement en dialogue avec le monde réussit-elle son travail de recontextualisation devant les situations nouvelles d'aujourd'hui? L'auteur esquisse trois stratégies contemporaines de la théologie: une rethéologisation du monde sacrifiant l'autonomie de ce dernier, une corrélation à tous crins risquant la perte de la dimension théologale et finalement une interruption saisie comme une recontextualisation de la corrélation en contexte postmoderne.
Tous droits réservés (c) Faculté de théologie et de sciences des religions, Université de Montréal, 2006
Ce document est protégé par la loi sur le droit d'auteur. L'utilisation des services d'Érudit (y compris la reproduction) est assujettie à sa politique d'utilisation que vous pouvez consulter en ligne.

https://apropos.erudit.org/fr/usagers/politique-dutilisation/ 


\title{
L'expérience de la théologie: corrélation, interruption et recontextualisation
}

\author{
Marc DUMAS \\ Faculté de théologie, d'éthique et de philosophie \\ Université de Sherbrooke
}

Les responsables du présent numéro ont circonscrit trois lieux privilégiés où la théologie est remise en question: l'université, l'Église et la société. $\mathrm{Ne}$ devrait-on pas tout d'abord évacuer la théologie de l'université publique? Qui dit théologie suppose une orientation confessionnelle particulière, une adhésion à un corpus dogmatique, etc. Cela ne contrevient-il pas à la mission de l'université, qui favoriserait ces croyants-ci et non ceux-là ? Devraiton accueillir à l'université des facultés orthodoxes, protestantes, voire juives, musulmanes, etc. ? Ou ne devrait-on pas plutôt, devant cette inflation possible de facultés, tout remplacer par une seule faculté de sciences religieuses plus neutre et libre de toutes les possibles pressions confessionnelles ${ }^{1}$ ?

Les instances ecclésiales n'auraient-elles pas aussi raison de se méfier d'une théologie trop distante de leur héritage et de leurs projets? Les travaux des théologiennes et des théologiens doivent-ils simplement se satisfaire d'être un appui organique, c'est-à-dire de recevoir, d'organiser et de présenter le plus clairement possible le matériel institutionnel, sans dire mot, sans poser le regard critique attendu par nos contemporains et nos sociétés? Leurs travaux ne doivent-ils pas, à l'inverse, être à la fois accueillants et critiques des lectures institutionnelles proposées? Tantôt inscrits dans la Tradition, tantôt sensibles à la culture, ces théologiennes ou théologiens collaborent à une meilleure intelligence de la foi pour la communauté et la société, auxquelles elles ou ils appartiennent.

1. Voir à ce propos le numéro thématique de Studia canonica, 37/2 (2003).

(C) Revue Théologiques 2006. Tout droit réservé. 
Et dans le domaine social et culturel, le déclin des institutions religieuses d'une part et le retour du religieux d'autre part ne justifieraient-ils pas l'interrogation sur la pertinence non seulement de la théologie, mais aussi du patrimoine chrétien tout court (voir Hoping et Arens 2000) ? La théologie devrait-elle alors se taire devant l'émergence d'un religieux peu ou non institué, moins organique et pourtant interpellant pour les contemporains? Il en va du dynamisme de la théologie d'être ouverte et à l'écoute de ce religieux actuel, de ces bricolages spirituels, pluriels, étranges, mais néanmoins signifiants pour plusieurs de nos contemporains. Nous sommes réunis pour discuter de ces questions importantes, du moins invités à y réfléchir ici le plus sérieusement possible.

Cet exposé veut modestement contribuer à cette réflexion. Il s'agit moins pour moi de présenter mes recherches sur une possible théologie en expérience, mais plutôt de considérer l'expérience de la théologie, qui s'efforce de poursuivre le dialogue avec le monde contemporain (voir un témoignage de ce dialogue dans Moltmann 1999). Comment se déplace ce réflexe dialogal dans un contexte nouveau, différent, plus près de nous et marqué par l'aujourd'hui ? Cette proximité est stimulante, car elle donne l'impression de prendre part aux travaux théologiques qui pourraient être nécessaires aux contemporains; elle est aussi risquée, car la distance faisant défaut, le discernement est à reprendre sans cesse. Mais si nous voulons faire une théologie transgressive, qui ouvre un chemin neuf pour aujourd'hui, nous devons oser et prendre ce risque. Comment la théologie se lie-t-elle au monde ? Réussit-elle à discerner comment se positionner face aux nouvelles sensibilités caractéristiques de la culture? Le déplacement contemporain entraîne nécessairement un effort de recontextualisation de la théologie, si on veut précisément illustrer sa pertinence et dissiper les questions embarrassantes dont elle est l'objet tant par l'université que par la culture, la société et même l'Église, avec laquelle la théologie devrait entretenir un rapport particulier. Comment transgresser l'embarras général esquissé ci-dessus et ouvrir un chemin qui «colle» aux situations nouvelles d'aujourd'hui ?

Je procéderai par quelques remarques qui pourraient fournir les balises d'un chemin, d'un faire théologique contemporain. Ce sera une première évaluation fragmentaire des efforts de recontextualisation de certains théologiens, afin d'en dégager les mérites et les limites. Et si je voulais préciser mon intitulé, je l'énoncerais comme suit : l'expérience de la théologie: l'interruption comme recontextualisation de la corrélation en postmodernité. Notez que je prends ce dernier terme avec des pincettes. Devant les déplacements du religieux, la pratique théologique se déplace aussi. Voyons voir pourquoi et comment. 


\section{L'expérience de la théologie}

Une première remarque désire clarifier l'expression «l'expérience de la théologie », car celle-ci peut porter à confusion. Elle renvoie à la sagesse acquise de cette science millénaire, à ce qu'elle traverse et a traversé dans les temps et les espaces. Elle est une praxis, un acte, un faire, et elle manifeste une volonté de poursuivre ses expériences à travers les travaux et les outils développés par les théologiennes et les théologiens. L'expérience de la théologie peut être ancestrale, riche, imposante, possiblement assise sur des acquis et être dogmatique; elle peut être, au contraire, située, fragile, risquée, limitée, ouverte à collaborer aux constructions des contemporains, lorsqu'il s'agit de se situer dans les horizons du sens, dans les irruptions religieuses ou dans les courants spirituels, dans les rencontres des grandes religions ou encore dans la pluralité et la diversité sociétales. Il s'agit peut-être d'un pas en arrière pour prendre conscience des enjeux et des chemins des théologies d'aujourd'hui. Sont-elles académiques? ecclésiales? sociales? Assurément, mais non comme on le voudrait. La théologie ouvre à une intelligence différente de celle des sciences humaines. Elle critique ce qui empêche l'Église d'annoncer la Bonne Nouvelle. Elle s'inscrit dans la société, comme ferment de transformation qui contribue à un "rendre compte de la foi " pertinent pour les humains. Bref, la théologie est en porte-à-faux face aux attentes, car elle a la fonction de donner un éclairage différent à l'université, l'Église et la société. Il devient donc nécessaire de déployer des stratégies conceptuelles pour inscrire le possible apport de la théologie et de la foi chrétiennes aux situations contemporaines souvent lues simplement sous les modes de la catastrophe, de la panique et de la détresse; et si la théologie permettait de passer de la catastrophe à la crise, de la panique à la confiance et de la détresse à l'espérance?

\section{Le travail théologique}

Une seconde remarque touche la geste relationnelle du travail théologique à travers les âges. On cherche à mieux expliciter par la raison la foi de la communauté croyante, mais on doit aussi être apte à rendre compte de sa foi pour le monde dans lequel les croyants vivent. Le réflexe d'intelligence de la foi usera des outils de l'époque et reprendra sans cesse au fil du temps et avec une créativité extraordinaire la matière de foi. Je devrais ajouter à la matière de foi l'acte de foi, le fait que la foi réponde à une présence théologale au cœur du monde. Il existe un rapport intrinsèque entre la forme de la 
tradition chrétienne d'une époque et le contexte historique qui soutient le travail théologique de cette époque. Et en dépit des changements de la tradition à travers les âges, une certaine identité ou référence à la foi perdure. Elle est permise par un événement, par un récit particulier (voir Certeau 1971). Et même si les conditions du dialogue entre la foi et le contexte peuvent changer, le dialogue demeure une nécessité incontournable méthodologiquement. Cette exigence méthodologique du dialogue ne doit pas être gommée; elle risquerait précisément de soulever la question de la pertinence de ce numéro, si on oubliait et gommait la nécessité de la relation entre la foi, la tradition et la théologie d'une part, et la culture et la société d'autre part.

\section{La corrélation}

La troisième remarque évalue les théologies des $\mathrm{XIX}^{\mathrm{e}}$ et $\mathrm{XX}^{\mathrm{e}}$ siècles qui, en contexte de modernité, insistent sur un principe de continuité entre la théologie et la culture. Le mode de recontextualisation de la foi chrétienne s'adosse aux différents processus socioculturels de la modernisation (un certain emploi de la rationalité, un principe d'autonomie et un travail de libération sociale) pour y inscrire les lieux d'action du Dieu sauveur. Les critères épistémologiques modernes (universalité, transparence et communicabilité) sont intégrés dans les réflexes théologiques, et les théologies de la corrélation articulent la médiation entre la foi et la culture. Développée de façon magistrale surtout par Paul Tillich, la méthode corrélative insiste sur la continuité, l'harmonie et un chevauchement important de la foi et de la culture. Cette méthode sera toutefois reprise et améliorée par plusieurs théologiens de la seconde moitié du $\mathrm{XX}^{\mathrm{e}}$ siècle, car bien que prometteuse, elle avait ses ratés (voir Dumas 2004a). Elle ne donne plus les fruits attendus. Elle bute sur l'épuisement de la thèse de la sécularisation, qui est même qualifiée de mythique par ses plus ardents promoteurs d'il y a plus ou moins trente ans (voir par ex. Berger 1999). Sa plausibilité, qui reposait sur la théorie de la somme zéro (plus de sécularisation conduit à moins de religion; à l'inverse, moins de sécularisation conduit à plus de religion), est contestée devant le vaste renouveau religieux. On se rend bien compte que la religion survit à la modernisation. Une situation nouvelle émerge et elle est l'objet d'une quatrième remarque.

\section{La situation postmoderne}

Cette remarque porte sur cette situation nouvelle, dite postmoderne. Notons que les analyses pour mieux la qualifier et la distinguer de la modernité se 
poursuivent. Nous sommes dans ce bouleversement qui se manifeste par des critiques envers la modernité et par de nouvelles sensibilités épistémologiques qui conduisent la théologie à se recontextualiser.

Au lieu de parler de sécularisation, parlons plutôt de désinstitutionnalisation, c'est-à-dire de détraditionnalisation, d'individualisation et de pluralisation. Ces sensibilités sont validées à travers le caractère polymorphe, pluriel, pluriforme du religieux et de la vie chrétienne elle-même, mais aussi à travers les différentes visions du monde et les multiples styles de vie. Cela rend très difficile d'affirmer dans ce nouveau contexte l'existence d'un dialogue entre la foi et la culture. Le travail dialogal risque davantage d'être conflictuel et ardu, car les partenaires sont pluriels : il y a des fois, des théologies, des cultures, etc.

Cette situation postmoderne met en valeur des sensibilités et des réflexes que la posture moderne avait éliminés soit en les intégrant, soit en les excluant. La radicalité de l'hétérogénéité résiste à un discours hégémonique qui nivelle la différence de l'autre. On met en valeur la contingence des constructions de sens, leurs limites, leur caractère situé et particulier. Ces sensibilités à l'autre et à la différence critiquent la tendance totalisante à conclure facilement au consensus et à l'harmonisation. Dans ce nouveau contexte, la position de pur observateur est remise en cause et un espace est donné aux participants qui sont en relation, en confrontation, en conflit; ceci exige de considérer la discontinuité dans le dialogue.

Dans ce contexte, le récit chrétien devient un récit parmi d'autres; il n'est plus un grand récit marqueur de notre société. Le récit chrétien ne va plus de soi comme auparavant; il s'effiloche dans les consciences; la culture religieuse chrétienne des contemporains s'estompe. La foi chrétienne est alors mise au défi par une culture postchrétienne et plurielle, qui peut remettre en question l'identité spécifique des croyants. L'engouement pour la pluralité religieuse, les grandes religions et les différents styles de vie contribue à cette remise en question de l'identité des chrétiens et des chrétiennes.

\section{Les trois stratégies théologiques actuelles}

La cinquième remarque porte sur les stratégies théologiques en postmodernité. J'identifie trois stratégies. Les deux premières sont au risque et au détriment soit du dialogue avec le contexte, soit du caractère propre du travail théologique. La première stratégie s'efforce de rethéologiser le monde. Le contexte de sécularité, constitutif de l'humain et de son monde en modernité, est abandonné et le monde est saisi comme négatif et aliénant. 
Son oubli de Dieu conduit à un dépassement, mieux à un repli ou un retour qui a comme objet une concentration originale et antérieure de la relation à Dieu, précédant les développements du théisme et de l'athéisme modernes. Ici, la rupture radicale avec le contexte est privilégiée, puisque le don de Dieu ou son espace sont ailleurs. On peut penser à des mouvements théologiques qui s'inscrivent dans ce rejet du monde pour faire théologie autrement ${ }^{2}$.

La seconde stratégie s'inscrit dans l'horizon moderne, qui est perçu comme quelque chose de positif. Cet horizon a permis à la théologie de se poser comme un projet corrélatif entre la tradition et la modernité. Dans cette stratégie, on fait, bien conscient des difficultés de la corrélation dans le nouveau contexte, des aménagements en usant par exemple d'un modèle corrélatif ravalé et en dogmatisant à la limite une continuité irréductible entre l'humain et le chrétien, entre l'anthropologie et la théologie. L'insistance trop unilatérale sur la continuité entre l'anthropos et le théos ne risque-t-elle pas d'occulter le sens propre de la théologie, de fonctionnaliser tellement le caractère anthropologique que la dimension théologale ne peut apparaître que comme un redoublement, une couleur chrétienne à un symbole, un rite ou encore un récit ? L'effort de poursuivre à tout prix la corrélation occulte la dimension spécifique de la foi chrétienne et elle devient comme un exemple ou une couleur, un cadre possible de gestion thérapeutique ou encore une fonction en vue de favoriser la croissance et la coexistence humaines. La dimension théologale est oubliée.

En effet, combien de travaux théologiques se réduisent à des lectures sociologiques, psychologiques ou historiques. Si ces travaux sont éclairants pour saisir les horizons anthropologiques des contemporains, ils ne marquent pas toujours comment le théologal s'inscrit dans les plis et replis de l'histoire, de l'humain et de la société; ils ne marquent pas toujours comment cette inscription ébranle et transforme les différentes lectures ${ }^{3}$.

La troisième stratégie de recontextualisation théologique est celle de l'interruption. Il faut nous pencher plus à fond sur cette avenue de l'exercice théologique pour aujourd'hui car, avec elle, on prétend pouvoir mieux articuler en contexte postmoderne, la manière dont il serait possible de faire une expérience de la théologie signifiante et pertinente. Je développe ce point à l'aide des différents travaux de Lieven Boeve, qui a passablement publié sur ce thème ces dernières années (voir 2003a; 2003b). Il a

2. Voir par exemple le mouvement théologique de Radical Orthodoxy (Milbank, Pickstock et Ward 1999; Pabst et Venard 2004).

3. Voir ce type de constat avec la thématique de l'expérience dans Dumas 2004b. 
assez bien cristallisé une tendance qui intéresse les méthodes corrélatives modernes, à inscrire dans le contexte nouveau la geste dialogale de la théologie avec le monde, la culture et la société. Comment dépasser la remise en question de l'identité spécifique de la foi chrétienne et dialoguer avec une culture, dont les contours sont pluriels, situés, complexes, sans tomber dans un relativisme culturel, pire, sans oublier la dimension théologale spécifique de la foi chrétienne? La thèse de Boeve est présentée dans une double perspective quant il s'agit de répondre à ces questions. La perspective ad extra et celle ad intra. On pourrait parler de mouvements ou de réflexes suscités par le dialogue entre la foi et le contexte.

La première perspective (ad extra) rencontre le contexte, l'altérité des autres, et ouvre les yeux sur la particularité irréductible du récit chrétien et sur la difficulté à le dire aujourd'hui. On nous croirait en perte de notre particularité; on dirait qu'un langage s'estompe pour dire Dieu (espace symbolique, rituel, horizon herméneutique flou). L'apprentissage de la langue ou du jeu de langage se fait difficilement, l'expérience même de Dieu dans l'horizon de foi devient presque impossible. La perte de connaissance de la densité et de l'épaisseur du récit chrétien en conduit plusieurs à rechercher des structures analogues et des dénominateurs communs. Mais cette recherche d'air de famille ne peut occulter le caractère propre et les distinctions, les personnalités de chacun. Ainsi, par exemple, les religions monothéistes, qui confessent un seul Dieu, ont chacune des manières particulières d'évoquer Dieu.

La seconde perspective (ad intra), mise au défi par la pluralité des particularités, examine la tradition chrétienne en vue d'une recontextualisation. La pluralité invite à chercher une nouvelle relation au contexte, à se recontextualiser. Cet effort est inévitable. Dans cette seconde perspective, le jeu de langage de la tradition est modifié et s'enrichit d'une nouvelle recontextualisation.

L'interruption apparaît ici la clé de la rencontre et du dialogue. L'interruption par une altérité irréductible ne pourrait-elle pas être un lieu théologique, un lieu où apercevoir les traces de Dieu ? Ne serait-il pas envisageable de considérer l'Autre interrompant nos récits à travers la rencontre et la confrontation avec les autres? Une herméneutique interrompue demeure infinie et ouverte; elle ne se crispe pas, ne se sclérose pas, ni ne glisse dans une attitude fondamentaliste. La rencontre interruptive de l'altérité irréductible évite d'absolutiser la particularité, une absolutisation qui risquerait un braquage contre la contextualité ou encore un rejet d'une nouvelle expérience d'être chrétien. Cette rencontre interruptive évite aussi 
la réduction du discours chrétien au niveau des structures humaines générales. La différence se trouve dans la similarité. La pertinence du récit chrétien est proportionnelle à son identité, à son aptitude à se recontextualiser dans un contexte marqué par la pluralité. Elle évite enfin toute confusion et récupération d'expériences religieuses ambiguës sans perspective critique. Alors qu'on s'imagine accélérer le moteur de la recontextualisation, on s'adapte mal au contexte et on fait dérailler la dynamique.

Les deux perspectives présentées par Boeve participent à l'effort de recontextualisation de l'Évangile. Confrontées à l'altérité de l'autre, à la pluralité, à la faillite ou à la critique postmoderne des grands récits qui instrumentalisent, les sensibilités nouvelles travaillent cet effort de recontextualisation. La pluralité radicale développe une sensibilité pour la particularité irréductible du récit chrétien. L'altérité de l'autre avec qui je dialogue et qui m'interrompt m'oblige à modifier la relation de la tradition au contexte.

Les perspectives méthodologiques et stratégiques dans ce nouveau contexte passent donc par deux éléments pour la théologie. Premièrement, par la poursuite du dialogue. Celui-ci se fait tout d'abord en distinguant un mouvement ad extra et un ad intra, afin de clarifier comment la rencontre du monde conduit à redécouvrir, au cœur du pluriel, la particularité irréductible de la foi et comment l'examen de ce nouveau contexte a des conséquences pour le développement de sa propre narrativité; cet examen confrontant agit comme moteur de recontextualisation théologique. Deuxièmement, il ne faut pas oublier que quelque chose interrompt le récit, le mien comme celui des autres. L'altérité révèle les limites de la position.

\section{S'inquiéter du théologal ici et maintenant}

Concluons en revenant sur l'exposé d'ouverture du professeur Jean-Claude Petit. Moi non plus, je ne connais pas l'avenir de la théologie. Mais je crois fermement qu'elle en aura un si nous exerçons le travail théologique en n'oubliant pas le contexte où nous nous trouvons et surtout en n'oubliant pas que le caractère proprement théologique de nos activités est de s'inquiéter du théologal. L'interruption apparaît comme une corrélation postmoderne entre un contexte pluriel, un récit ouvert par Dieu et des traces d'un Dieu interrupteur de récits, de contextes, d'Églises malheureusement trop souvent en mode d'enfermement, d'exclusion. L'interruption cherche à articuler un équilibre entre la continuité et la discontinuité et à nous garder en mode d'ouverture, en mode d'expérience. Et dans leur épistémologie 
propre, d'autres, comme Michel de Certeau avec la rupture instauratrice, Tillich avec sa notion d'irruption, mais aussi les auteurs spirituels et mystiques, les auteurs des écrits mystagogiques, les auteurs des pages des deux Testaments, se sont inquiétés du théologal, chacun dans son contexte particulier. Il nous appartient de le faire ici et maintenant.

\section{Références}

Berger, P. (1999), The Desecularization of the World: Resurgent Religion and World Politics, Grand Rapids / Cambridge, Eerdmans.

Boeve, L. (2003a), Interrupting Tradition. An Essay on Christian Faith in a Postmodern Context, Louvain / Dudley, Peeters (Louvain Theological \& Pastoral Monographs 30).

- (2003b), «La définition la plus courte de la religion: interruption », Vie consacrée, 75 , p. 10-36.

Certeau, M. de (1971), «La rupture instauratrice ou le christianisme dans la culture contemporaine ", Esprit, 39, p. 1177-1214; repris dans M. DE Certeau, La faiblesse de croire, Paris, Seuil, 1987, p. 183-226.

Dumas, M. (2004a), "Corrélations d'expériences? ", Laval théologique et philosophique, 60, p. 317-334.

- (2004b), «Introduction à l'expérience en théologie. Pluralité ambiguité et nécessité », dans M. Dumas, F. Nault et L. Pelletier, dir., Théologie et Culture. Hommages à Jean Richard, Québec, Les Presses de l’Université Laval, p. 123-142.

Hoping, H. et E. Arens (2000), Wieviel Theologie verträgt die Öffentlichkeit?, Fribourg / Bâle / Vienne, Herder.

Milbank, J., C. Pickstock et G. Ward (1999), Radical Orthodoxy, Londres / New York, Routledge.

Moltmann, J. (1999), Erfahrungen theologischen Denkens. Wege und Formen christlicher Theologie, Gütersloh, Kaiser Gütersloher Verlagshaus.

Pabst, A. et O.T. Venard (2004), Radical Orthodoxy. Pour une révolution théologique, Genève, Ad Solem. 


\section{Résumé}

L'auteur s'interroge sur la remise en question de la théologie dans ses lieux, qui lui étaient habituellement acquis. En effet, l'université, l'Église et la culture semblent embarrassées par la théologie. Mais cette dernière historiquement en dialogue avec le monde réussit-elle son travail de recontextualisation devant les situations nouvelles d'aujourd'hui ? L'auteur esquisse trois stratégies contemporaines de la théologie: une rethéologisation du monde sacrifiant l'autonomie de ce dernier, une corrélation à tous crins risquant la perte de la dimension théologale et finalement une interruption saisie comme une recontextualisation de la corrélation en contexte postmoderne.

\section{Abstract}

The author wonders about the established premises of theology being called into question. The University, the Church and the Culture seem indeed embarrassed by its theology. But then again we may question if the latter, being in dialogue with the world through history, is really succeeding its recontextualization regarding today's new circumstances. The author outlines three contemporary theological strategies: a retheologization of the world sacrificing thereby the latter's autonomy, a fiery correlation risking to lose the theological dimension and finally an interruption understood as a recontextualization of the correlation in a postmodern context. 